\title{
Vocabulary Facilitation on Technical Modules for ESL Learners: A Case Study of a Sri Lankan Higher Educational Institute
}

\author{
Chathurika Senevirathna ${ }^{1}$, Shashitha Jayakody ${ }^{2, *}$ \& H Rangika Iroshani Peiris ${ }^{2}$ \\ ${ }^{1}$ English Language Teaching Unit, Sri Lanka Institute of Information Technology, Sri Lanka \\ ${ }^{2}$ Department of Business Management, Faculty of Business, Sri Lanka Institute of Information Technology, Sri \\ Lanka \\ *Correspondence: Department of Business Management, Faculty of Business, Sri Lanka Institute of Information \\ Technology, New Kandy Road, Malabe, Sri Lanka. E-mail: s.g.jayakody@gmail.com
}

Received: December 14, 2016

Accepted: December 19, 2016 Online Published: December 27, 2016

doi:10.5430/wjel.v6n4p1

URL: http://dx.doi.org/10.5430/wjel.v6n4p1

\begin{abstract}
This study focuses on the degree of facilitation of the English language module on a technical module offered for a degree program in a higher educational institute in Sri Lanka. The sample consists of 5,855 words from one technical module in the stream of Accounting and Finance and 10,554 words from one English language module prescribed for the BBA (Special) Degree program during the first year first semester of undergraduates. The level of facilitation was measured in terms of vocabulary; an essential component found in the empirical literature to acquire the technical knowledge in tertiary education. In order to achieve the main objective, "whether or not the language module facilitates the technical module" the researchers utilized Academic Word List (AWL) and examined the presence of AWL items in both modules and compared the common distribution of AWL items. The results showed 12.33\% presence of AWL items in the technical module and 3.95\% in the language module. 65 AWL word families were identified as common to both modules. The facilitation of the language module on the technical module in terms of vocabulary is $42.20 \%$. Interestingly, the most frequently used 10 AWL items are not common to both modules. Collocation and gap making can be suggested as appropriate vocabulary activities in order to enhance the exposure of the ESL learners to vocabulary.
\end{abstract}

Keywords: The Academic Word List (AWL); AWL coverage; facilitation; vocabulary; technical module

\section{Introduction}

There is a growing interest about using English as the accepted medium of instruction in Sri Lankan higher educational system. However, this phenomenon triggers many social and research discussions regarding students' academic adaptability in ESL context. Previous researchers looked into core components of language in relation to achieving academic goals. It is in this background that vocabulary teaching has earned tremendous attention worldwide in ESL research and ELT pedagogy. The studies carried out by Li and Qian (2010), Wang, Liang \& Ge (2008), Mudraya (2006), Coniam (1999), show the importance of vocabulary building in several disciplines with the aid of AWL. Similarly in the Sri Lankan context, studies of Illangakoon (2012), Kumara (2009) and Perera (2006) highlight the significance of English vocabulary building in the teaching and learning process of higher education.

The current study observes the degree of facilitation of the English module towards the selected technical module in terms of vocabulary. Creating academic word list has dialectically evolved assuring AWL developed by Coxhead (2000) still as a centralized research source. It is this backdrop that drove the researchers to utilize AWL as an important yardstick to assist the present case study.

This study is an attempt to examine whether the prescribed English language teaching modules facilitate the compulsory technical modules offered for the BBA (Special) degree program in the Faculty of Business, at Sri Lanka Institute of Information Technology. This case study specifically focuses on the first year first semester technical module, "Fundamentals of Accounting" (FA) and the English language module "English Language Skills I" (ELS1). 


\subsection{Importance of Vocabulary}

Importance of vocabulary for ESL learners is observed in different aspects. Shaw (1991) finds that the most prominent language problem of both native and non-native learners in their academic learning and writing is vocabulary. Especially in the university system vocabulary can be of paramount assistance for students to acquire the essential technical knowledge. Li and Pemberton (1994) state that it is not discipline specific technical vocabulary that tertiary students find difficult in their learning process, but "the vocabulary with a middle-frequency of occurrence across the texts of various disciplines that students find most problematic (p.184).”

\subsection{Needfulness of AWL in ESL Pedagogy}

It is in this context that needfulness of AWL is proved important in the ESL pedagogy. To quote Coxhead (2000), "the AWL shows learners with academic goals which words are most worth studying (p.1)." Vongpumivitch, Huang \& Chang (2009) emphasize "the importance of learning the frequently-occurring AWL words, especially those that come from Coxhead's first two sublists, regardless of the learners' filed." Hence, our research uses AWL as a foil to address the research objectives. AWL is used to examine the common distribution of the academic words in the two modules with the objective of finding out whether or not the ELS module serves as a facilitator module to FA in terms of vocabulary.

\subsection{The Academic Word List}

Compilation of word lists dates back to 1970's (Note 1). However the most eminent word list in the academia is the list created by Coxhead (2000). This consists of 570 word families from the disciplines Arts, Commerce Law and Science which is based on a 3.5 million word corpus. The coverage of the AWL word list in the stream of commerce is $12 \%$ (Coxhead, 2000). The AWL is grouped into ten sublists depending on frequency. Except the tenth sublist, which has 30 word families the rest contain 60 word families each.

\section{Objectives}

The main objective of the study is to investigate whether or not the language module "ELS1" facilitates the technical module "FA" in terms of vocabulary. In order to achieve the primary objective we have three research questions.

1) What is the presence of AWL items in the FA module?

2) What is the presence of AWL items in the ELS 1 module?

3) What is the common coverage of AWL items in both modules?

\section{Methodology}

\subsection{Sample}

Prescribed teaching material for the BBA (Special) degree program at the Faculty of Business, at Sri Lanka Institute of Information Technology was considered for the study. The BBA (Special) degree program consists of five main specializing streams (Note 2). The informal interviews with undergraduates and the subject specific lecturers revealed that the difficulty of medium is a common barrier to acquire the required technical knowledge of the particular modules. Therefore, we felt the need of considering the vocabulary items in the prescribed technical modules. This case study limits only to the area of "Accounting and Finance". The undergraduates are first exposed to the subject "Accounting and Finance" during their first year through the module "Fundamentals of Accounting". Thus we selected FA as our technical module.

On completion of General Certificate of Education - Advanced Level (Note 3) examination, students from different socio economic backgrounds enter the degree program which is totally conducted in English. Majority of the students complete their secondary education in their first language. In this event, it is Sinhala or Tamil. Thus the degree program would be the first experience of the majority in following technical subjects in the medium of English. Thus the first year first semester modules were found crucial in orienting them to learning process of the university education. Therefore we concentrated on first year first semester modules.

There are two English language modules for the degree program. Both modules are taught using the prescribed English language text books "English Language Skills 1" and "English Language Skills 2". Both text books are taught during first year in first and second semesters respectively.

"English Language Skills 1" is a text book compiled by the in house staff at the institute with the objective of 
enhancing the primary language skills of first year students' (Note 4). The text book largely contains lessons from General English. Fundamentals of Accounting module is comprised of presentation slides based on the module outline prepared by the in-house staff (Note 5).

The teaching materials of both modules were standardized according to the method employed by Chen and Ge (2007). We eliminated items which cannot be counted such as diagrams, charts, numbers and references. After the standardization procedure our text consisted of 5,855 words from FA and 10,554 words from ELS1.

\subsection{Method}

In order to achieve the objectives of the current study we utilize the online software program developed by Tom Cobb, the Compleat Lexical Tutor (CLT). This can be accessed free of charge on http://www.lextutor.ca/. The site consists of various features which enables to carry out lexical analyses (Note 6). The "VocabProfiler" feature in the software breaks down the uploaded text into three lists, namely 'first thousand frequent words' (K1), 'second thousand frequent words' (K2) both of which developed from the General Service List (GSL) and thirdly the Academic Word List. The output from this feature shows the coverage in selected text relative to the aforementioned three lists. Using this output we identified the coverage of AWL items in both FA and ELS1 modules. Further, we extracted the AWL families (head-words) which are present in the input text of both modules with the intention of mapping the technical module with the language module. The mapping of the head-words was carried out using Vlookup function in MS Excel.

\section{Results}

Table 1. Coverage of the FA Text Book Material by the GSL and the AWL

\begin{tabular}{clcccc}
\hline Word list Number & Word list description & Families & Types & Tokens & Percent \\
\hline 1 & K1 Words, 1-1000 & 405 & 627 & 4184 & $71.47 \%$ \\
2 & K2 words, 1001-2000 & 68 & 98 & 324 & $5.53 \%$ \\
3 & AWL words (Academic) & 154 & 237 & 722 & $12.33 \%$ \\
Not in the list & Off List words & & 172 & 624 & $10.66 \%$ \\
Total & & $627+$ & 1134 & 5854 & $100 \%$ \\
\hline
\end{tabular}

Table 1 shows the coverage of General Service List (GSL) which represents K1 and K2 and the Academic Word List (AWL) in the technical module FA. The results indicate that $89.33 \%$ of the tokens in the FA module represent a combination of the GSL and the AWL. The AWL coverage of the tokens in the selected module is $12.33 \%$.

This result justifies the statement made by Coxhead and Byrd (2007) that AWL "covers approximately $10 \%$ of any academic text”. The results can further be compared to the recent research findings of Li and Qian (2010) which covers $10.46 \%$ of their selected corpus. These researchres have profiled the presence of the AWL items in their financial corpus, with the objective of exploring ways to effectively teach the AWL items in the Hong Kong Financial Services Corpus. Our results further conform to the findings of Chen and Ge (2007) at their attempt to identify the AWL words in selected medical research articles as their findings manifest a coverage of $10.07 \%$. Moreover in the Sri Lankan context, Illangakoon (2012) reports similar findings (11.11\%) in her corpus based on five subjects in the stream of Arts. Contrastingly, a study based on a Chemistry corpus reveals only 9.6\% of AWL coverage which is lower than the percentages reported in the above studies (Valipouri \& Nassaji, 2013).

Table 2. Coverage of the ELS1 Text Book Material by the GSL and the AWL

\begin{tabular}{clcccc}
\hline Word list Number & Word list description & Families & Types & Tokens & Percent \\
\hline 1 & K1 Words, 1-1000 & 682 & 1128 & 8251 & $77.44 \%$ \\
2 & K2 words, 1001-2000 & 269 & 359 & 760 & $7.13 \%$ \\
3 & AWL words (Academic) & 186 & 237 & 421 & $3.95 \%$ \\
Not in the list & Off List words & & 714 & 1223 & $11.48 \%$ \\
Total & & $1137+$ & 2438 & 10655 & $100 \%$ \\
\hline
\end{tabular}


Table 2 shows the coverage of the language module, "ELS 1" by the GSL and the AWL. The total coverage in AWL is $3.95 \%$, when compared with the technical corpus-based studies, the above percentage stands lower than the percentage (10\%) reported by Coxhead and Byrd (2007). However, our findings are in consistence with the results of Dang and Webb (2013) which shows a coverage of 4.41\% in their study based on academic spoken English.

Table 3. Distribution of AWL Word Families in ELS1 and FA

\begin{tabular}{lcccc}
\hline & \multicolumn{2}{c}{ ELS 1 } & \multicolumn{2}{c}{ FA } \\
\multicolumn{1}{c}{ Summary } & Counts & Percentage & Counts & Percentage \\
\hline AWL total word families & 570 & & 570 & \\
AWL families in the input text & 186 & $32.63 \%$ & 154 & $27.01 \%$ \\
AWL families not in the input text & 384 & $67.36 \%$ & 416 & $73.15 \%$ \\
\hline
\end{tabular}

Note: All the percentage values are rounded to two decimal places. These percentages do not refer to tokens in text but rather number of families. This analysis applies only to standard list items NOT any recognized proper nouns.

Table 3 shows the distribution of the total AWL word families in both modules. ELS 1 covers 32.63\% while FA covers 27.01\%. Interestingly, ELS 1 has a 5.62 of higher percentage difference. This distribution has further been illustrated graphically in Figure 1.

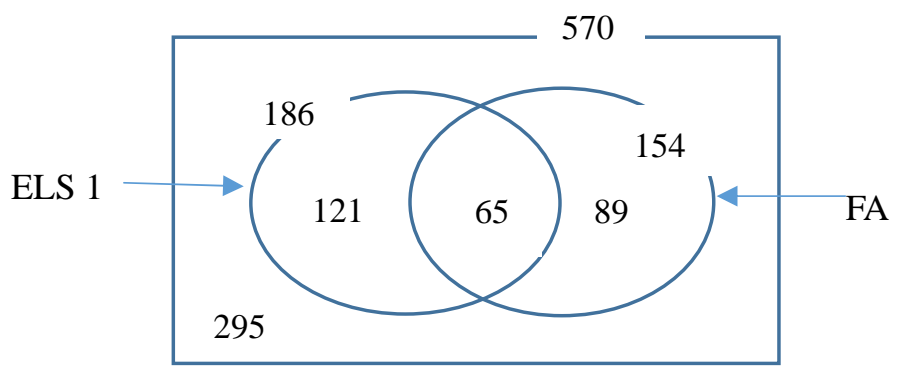

Figure 1. AWL Word Family Distribution

With reference to the third research question there are 65 AWL word families recorded common to both modules as shown in Figure 1. The remaining 89 word families in the FA module are not covered in the ELS 1 module. Thus these findings indicate that the degree of facilitation of the language module; ELS 1 on the technical module; FA is $42.20 \%$ in terms of vocabulary. The identified 65 common word families are illustrated in table 4 .

Table 4. Common AWL items in FA and ELS 1 Text

\begin{tabular}{lllll}
\hline Affect & Contract & Function & Method & Relevant \\
Approach & Correspond & Fund & Negate & Rely \\
Appropriate & Create & Generate & Neutral & Require \\
Assign & Define & Identify & Normal & Resource \\
Assist & Distribute & Indicate & Occur & Secure \\
Assume & Document & Individual & Option & Significant \\
Assure & Economy & Institute & Overall & Source \\
Available & Error & Internal & Percent & Specific \\
Benefit & Establish & Involve & Period & Task \\
Bias & External & Issue & Physical & Transfer \\
Chart & Factor & Job & Proceed & Trend \\
Communicate & Final & Margin & Professional & Vary \\
Consent & Format & Maximize & Purchase & Version \\
\hline
\end{tabular}


Figure 2 is an illustration of comparison in the distribution of AWL sublists in both modules. The coverage of AWL sublists in Coxheads' corpus manifests a decreasing trend (Coxhead, 2000). Vongpumivitch et al. (2009) have also observed the same pattern. However in the selected modules in the present study the overall pattern follows the similar decreasing shape of the distribution with minor differences in individual sublists.

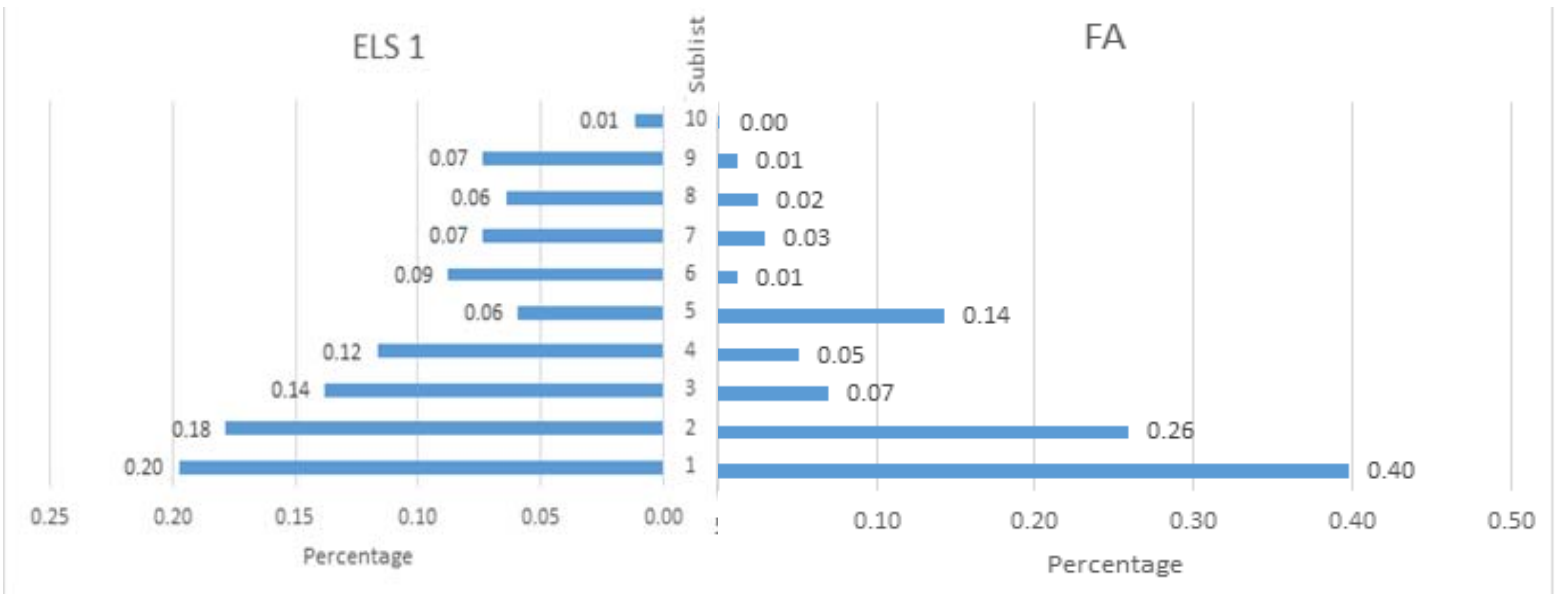

Figure 2. Comparison of subsists

Further we extended our study to examine the frequency of AWL items in both modules to identify how frequently the both modules expose the ESL learners to the top 10 AWL items.

Table 5. AWL Items (Word Families) in the Modules

\begin{tabular}{cccccccc}
\hline \multicolumn{3}{c}{ ELS 1 } & \multicolumn{5}{c}{ FA } \\
\hline AWL item & Frequency & Item* \% & Cumulative \% & AWL Item & frequency & Item \% & Cumulative \% \\
\hline document & 12 & 0.03 & 2.85 & finance & 70 & 0.10 & 9.70 \\
format & 12 & 0.03 & 5.70 & credit & 50 & 0.07 & 16.62 \\
transport & 12 & 0.03 & 8.55 & ratio & 36 & 0.05 & 21.61 \\
appropriate & 11 & 0.03 & 11.16 & purchase & 33 & 0.05 & 26.18 \\
insert & 10 & 0.02 & 13.54 & journal & 21 & 0.03 & 29.09 \\
compute & 9 & 0.02 & 15.68 & period & 20 & 0.03 & 31.86 \\
job & 9 & 0.02 & 17.81 & income & 19 & 0.03 & 34.49 \\
confer & 7 & 0.02 & 19.48 & item & 18 & 0.02 & 36.98 \\
function & 7 & 0.02 & 21.14 & entity & 16 & 0.02 & 39.20 \\
paragraph & 7 & 0.02 & 22.80 & factor & 15 & 0.02 & 41.27 \\
\hline
\end{tabular}

*Item \% = frequency of the specific word family / the frequency of all the AWL items in the module.

Table 5 shows the top 10 AWL items in ELS 1 and FA modules. ELS 1 has a cumulative coverage of 22.80\%. FA has a cumulative coverage of $41.27 \%$. It is nearly twice the size of ELS1. Interestingly, none of the top 10 AWL items in each module are shared mutually. Considering the 65 common items (Table 4), it is 5 from the top 10 AWL items in ELS 1 module and 3 from the FA module. The findings further reveal that the learners are exposed to 8 common word families from the above identified high frequency word families.

\section{Conclusion}

In our study to identify the degree of facilitation of the ELS 1 module on the FA module, we first profiled the 
presence of AWL items in each module. The results show that the presence of the AWL items in the FA module is $12.33 \%$ and in the ELS 1 module it is 3.95\%. These results adhere to the previous literature (Li \& Qian, (2010), Chen \& Ge, (2007), Illangakoon, (2012), Dang \& Webb, (2014)). Based on the findings of the first and second research questions we measured the degree of facilitation to answer the third research question by analyzing the coveage of AWL items in both modules. At this stage 65 items were identified as common to the technical module and the language module. Hence the facilitation of the ELS 1 module on the FA module in terms of vocabulary is $42.20 \%$. Regarding the frequency of AWL items in both modules, the most frequently used 10 AWL items in each module are not common to both.

\section{Pedagogical Suggestions}

Based on the result that 65 words in the technical module are facilitated by the language module, to enhance the exposure of the ESL learners further in terms of vocabulary, the following methods can be suggested. Collocations and Gap making would be appropriate vocabulary activities. This recommendation is made as the program AWL Gapmaker (Note 7) provides the facility to create gap filling exercises automatically according to the input. When preparing the input, high frequency items identified in the FA module can be considered.

Moreover teaching lexical chunks as suggested in the lexical approach is relevant here (Lewis, 1997). Vocabulary exercises can be designed to introduce certain words and lexical patterns through collocations. When outlining the activities, the 89 word families (Refer Figure 1) identified as not covered in the language module, can be given prominence.

\section{References}

Champion, M. E., \& Elley, W. B. (1971). An Academic vocabulary list. New Zealand Council for Educational Research.

Chen, Q., \& Ge, G. C. (2007). A Corpus-based lexical study on frequency and distributin of Coxhead's AWL word families in medical research articles (RAs). English for Specific Purposes 26(4), 502-514. https://doi.org/10.1016/j.esp.2007.04.003

Coniam, D. (1999). Second language proficiency and word frequency in English. Asian Journal of English Language Teaching, 9, 59-74.

Coxhead, A. (2000). A new academic word list. TESOL quarterly, 34(2), 213-238. https://doi.org/10.2307/3587951

Coxhead, A., \& Byrd, P. (2007). Preparing writing teachers to teach the vocabulary and grammer of academic prose. Journal of Second Language Writing, 16(3), 129-147. https://doi.org/10.1016/j.jslw.2007.07.002

Dang, T. N., \& Webb, S. (2014). The lexical profile of academic spoken English. English for Specific Purposes, 33, 66-76. https://doi.org/10.1016/j.esp.2013.08.001

Illangakoon, S. R. (2012). Identifying threshold vocabulary for academic study. Retrieved from digital.lib.ou.ac.lk

Kumara, M. (2009). Compilation and linguistic analysis of a dedicated corpus for the Applied Sciences. Research Symposium 2009 - Faculty of Graduate Studies. University of Kelaniya.

Lewis, M. (1997). Implementing the lexical approach: Putting theory into practice. Hove UK: Language Teaching Publications.

Li, E. S., \& Pemberton, R. (1994). An investigation of students' knowledge of academic and subtechnical vocabulary. In Proceedings joint seminar on corpus lingustics and lexicology, Guangzhou and Hong Kong. (Language Centre, HKUST, Hong Kong, 1994).

Li, Y., \& Qian, D. D. (2010). Profiling the Academic Word List (AWL) in a financial corpus. System , 38(3), 402-411. https://doi.org/10.1016/j.system.2010.06.015

Mudraya, O. (2006). Engineering English: A lexical frequency institutional model. English for Specific Purposes, 25(2), 235-256. https://doi.org/10.1016/j.esp.2005.05.002

Perera, K. (2006). Laying the foundations: Language planning in the ELTU. In English in the Multilingual Environment. Selected papers from the 3rd SLELTA international Conference. Colombo: SLELTA.

Praninskas, J. (1972). American university word list. Longman Group Limited. 
Shaw, P. (1991). Science research students' composing processes. English for Specific Purposes, 10(3), 189-206. https://doi.org/10.1016/0889-4906(91)90024-Q

Valipouri, L., \& Nassaji, H. (2013). A corpus-based study of academic vocabulary in chemistry research articles. Journal of English for Academic Purposes, 12(4), 248-263. https://doi.org/10.1016/j.jeap.2013.07.001

Vongpumivitch, V., Huang, J., \& Chang, Y. (2009). Frequency analysis of the words in the Academic Word List (AWL) ad non-AWL content words in applied lingustics research papers. English for Specific Purposes, 28(1), 33-41. https://doi.org/10.1016/j.esp.2008.08.003

Wang, J., Liang, S., \& Ge, G. (2008). Establishment of a medical academic word list. English for Specific Purposes, 27(4), 442-458. https://doi.org/10.1016/j.esp.2008.05.003

\section{Notes}

Note 1. Campion \& Elley (1971) and Praninskas (1972).

Note 2. Accounting and Finance, Human Capital Management, Marketing Management, Quality Management, Management Information Systems

Note 3. The final exam faced by the Sri Lankan students of free national education system.

Note 4. To quote the introduction of the text book. "The activities that have been built in, aim to give the learner practice in all four skills connected to learning a language".

Note 5. All subject module outlines taught for the degree program are approved by the University Grants Commission of Sri Lanka.

Note 6. The tool has been used by Illangakoon (2012) for her lexical analysis.

Note 7. http://www.nottingham.ac.uk/walzsh3/acvocab/awlgapmaker.htm 